\title{
Index autorum ad Vol. 164
}

\section{Confecit: C. Loeb-Schürch}

$(\mathrm{V})=$ Report - Vortrag - Communication $(\mathrm{B})=$ Book Reviews - Buchbesprechungen - Livres nouveaux

Auricchio, G., 228 Aust, W., 158 (B)

Ballantyne, A. J., 159 (B) Barishak, R., 199 Bartsch, C, 249 Bedrossian, E. H., 158 (B) Berry, 408 (B) Bihler, K., 154 (B) Binkhorst, C. D., 392 (V) Bleeker, G. M., 152 (B) Blümcke, S., 35 Bouman, M. A., 356 (V) Busacca, A., 156 (B) Butt, C, 284

Cambie, E., 81 Cherubini, T. D., 154 (B) Collier, M., 97

Delplace, M. P., 208 Denden, A., 84, 440 Deutman, A. F., 375 (V) Doden, W., 156 (B) Doerr, W., 152 (B) Donaldson, D. D., 78 (B) Draeger, J., 273 Duguid, 408 (B)

Erdei, Z., 71 Eugster, J., 337

Fatz, A., 150 (B) Fehér, J., 1,19 Fonda, G., 155 (B) Francois, J., 1,19, 81 Fritsche, P., 154 (B)

Gierhake, F. W., 155 (B) Gobin, M. H., 392 (V) Goder, G., 182 Goswami, A. P., 219

Hackelbusch, R., 273 Hagedoorn, A., 361 (V) Hammami, H., 422 Harders, H., 153 (B) Havener, W. H., 408 (B) Haye, C, 158 (B) Haymaker, W., 151 (B) Heine, 149 (B) Henkes, H. E., 385 (V) Hillemann, J., 321 Hoek, L. D. van, 355 (V) Hoover, R. E., 150 (B) Houtman, W. A., 405 (V) Huber, A., 464 (B) Hutschenreuter, K., 154 (B)

Kahán, I. L., 71 Kammann, J., 264 Karel, I., 161, 449 Keeney, A. H., 158 (B)

474

Index autorum ad Vol. 164

Keith Lyle, T., 152 (B)

Klethi, J., 128

Koliopoulos, J., 339

Kooijman, A. C, 398 (V), 402 (V)

Krejcí, L., 113

Langham, M., 152 (B) Lerche, W., 306 Lith, G. H. M. van, 385 (V) Littann, K. E., 84 Ludwig, H. H. H., 387 (V)

Madroszkiewicz, M., 236 Maione, M., 157 (B) Malbran, E., 50, 59 Martenet, A. C, 157 (B)

Martin-Doyle, J. L. C, 78 (B) Massaro, R. G., 387 (V) Michaelson, I. C, 159 (B)

Naumann, G., 293, 321 Niedorf, H. R., 35 Nover, A., 149 (B)

Offret, G., 158 (B) Oosterhuis, J. A., 362 (V) Otradovec, J., 449

Palimeris, G., 339 Papst, W., 252 Peleska, M., 161, 449 Pinckers, A., 137 Podestá, H. H., 249

Polack, F. M., 78 (B)

Quéré, M. A., 208

Rassow, B., 143 Ravault, M. P., 150 (B) Riehm, E., 249 Romano, A., 199 Rosengren, B., 409

Rubinstein, K., 362 (V) Ruckensteiner, E., 153 (B) Ruprecht, K. W., 293

Scouras, J., 422 Schramm, W., 155 (B) Smith, B., 154 (B) Starkiewicz, W., 156 (B) Stefani, C, 50, 59 Stein, R., 199 Stocker, F. W., 79 (B) Straub, W., 241, 408 (B) Streiff, E. B., 422 
Testa, M., 228 Thiel, H.-J., 35 Treister, G., 199

Ullerich, K., 264 Utermann, D., 284

Valu, L., 151 (B)

Van Hoek, L. D., v. Hoek, L. D. van

Van Lith, G. H. M., v. Lith, G. H. M.

van Veirs, E. R., 464 (B) Velhagen, K. H., 182 Velissaropoulos, P., 339

Waardenburg, P. J., 157 (B) Worst, J. G. F., 387 (V)

R. Radnót

Magda

122

Zuppinger

A.

$153(\mathrm{~B})$ 